\title{
The C-terminal rod 2 fragment of filamin A forms a compact structure that can be extended
}

\author{
Salla RUSKAMO*1, Robert GILBERT $\dagger$, Gregor HOFMANN $\dagger$, Pengju JIANG $\$$, lain D. CAMPBELL + , Jari YLÄNNE*2 and \\ Ulla PENTIKÄINEN *2 \\ ${ }^{*}$ Department of Biological and Environmental Science and Nanoscience Center, University of Jyväskylä, Jyväskylä, Finland, †Division of Structural Biology, University of Oxford, \\ Roosevelt Drive, Oxford OX3 7BN, U.K., †Department of Biochemistry, University of Oxford, South Parks Road, Oxford OX1 3QU, U.K., and §School of Pharmaceutical Engineering and \\ Life Science, Changzhou University, Changzhou, China
}

\begin{abstract}
Filamins are large proteins that cross-link actin filaments and connect to other cellular components. The C-terminal rod 2 region of FLNa (filamin A) mediates dimerization and interacts with several transmembrane receptors and intracellular signalling adaptors. SAXS (small-angle X-ray scattering) experiments were used to make a model of a six immunoglobulin-like domain fragment of the FLNa rod 2 (domains 16-21). This fragment had a surprising three-branched structural arrangement, where each branch was made of a tightly packed two-domain pair. Peptides derived from transmembrane receptors and intracellular signalling proteins induced a more open structure of the six domain fragment. Mutagenesis studies suggested that these changes are caused by peptides binding to the CD faces on domains 19
\end{abstract}

and 21 which displace the preceding domain A-strands (18 and 20 respectively), thus opening the individual domain pairs. A single particle cryo-EM map of a nine domain rod 2 fragment (domains 16-24), showed a relatively compact dimeric particle and confirmed the three-branched arrangement as well as the peptide-induced conformation changes. These findings reveal features of filamin structure that are important for its interactions and mechanical properties.

Key words: cytoskeletal cross-linking, cryo-EM, filamin, integrin binding, small-angle X-ray scattering (SAXS).

\section{INTRODUCTION}

Transmission of biochemical signals and mechanical forces across the plasma membrane is essential for the regulation of cell differentiation and proliferation [1,2]. This often requires connections between transmembrane adhesion receptors and the intracellular cytoskeleton. One of many proteins that connect F-actin (filamentous actin) to transmembrane receptors is FLN (filamin). FLNs are well positioned in cells to influence signal transmission as they have binding sites both for F-actin and the cytoplasmic tails or loops of membrane receptors, such as the $\beta$ chains of integrins, the major transmembrane adhesion receptors and G-protein-coupled receptors including DRs (dopamine receptors). FLNs also bind to various other cellular proteins including cytosolic signalling and adaptor proteins, e.g. migfilin [3-8]. Accordingly, through these interactions, FLNs are thought to bring together various proteins to enhance signal transmission [9].

The FLN protein family is encoded by three homologous genes [FLNA, FLNB and FLNC]. FLNa protein is the most abundant and widely expressed [4,5]. Each $280 \mathrm{kDa}$ FLN polypeptide contains an N-terminal actin-binding domain followed by 24 Ig (immunoglobulin)-like domains (FLN1-24) [5], with FLN24 mediating dimerization (Figure 1A) [10,11]. A flexible hinge region between domains 15 and 16 divides the chain of $24 \mathrm{Ig}$-like domains into rod 1 (FLN1-15) and rod 2 (16-24) (Figure 1A) [12]. Another hinge separates domains 23 and 24. Individual
FLN Ig domains are $\beta$-sandwich structures composed of eight $\beta$ strands. The domains in the rod 2 region have unusual interdomain interactions, with domains 16-17, 18-19 and 20-21 forming compact pairs (Figures 1B-1D) [13,14]. In the domain pairs $18-19$ and $20-21$, the $\beta$-strand $\mathrm{A}$ of domains 18 and 20 is folded together with the C- and D-strands of domains 19 and 21 respectively (CD faces of FLNa19 and FLNa21, Figures 1C and 1D). The same $C D$ face was earlier identified as a binding site for both integrins $[15,16]$ and migfilin $[17,18]$. The CD face of FLNa19 was also shown to be the binding site for DRD2 and DRD3 [19,20]. This kind of domain-domain interaction suggests that binding of FLN to some of its partners is autoinhibited. The exposure of these cryptic binding sites will require changes in conformation. These could be induced either by mechanical forces [21-23] or by binding to another interaction partner. In contrast with the 18-19 and 20-21 domain pairs, autoinhibition is not seen with the 16-17 pair (Figure 1B) [14]. The A-strand of domain 16 is unstructured and not associated with FLNa17 (Figure 1B); consequently the A-strand does not hinder an interaction partner, e.g. of platelet glycoprotein Ib $\alpha$, from binding to FLNa17 [14]. Domains FLNa22-23 in rod 2 also do not seem to exhibit compact domain packing [14]. Sequence comparison suggests that a similar pair-wise domain arrangement exists in FLNs throughout the animal kingdom, including Drosophila melanogaster (gene Cheerio) and Caenorhabditits elegans [14], although not in slime mold Dictyostelium discoideum [14]. Mutations in the C-terminal region of FLNs have been described in human patients and

Abbreviations used: DR, dopamine receptor; EM, electron microscopy; F-actin, filamentous actin; FLN, filamin; FSC, Fourier shell correlation; Ig, immunoglobulin; SAXS, small-angle X-ray scattering.

The cryo-EM reconstructions reported have been deposited in the European Bioinformatics Institute Electron Microscopy Database (http://www.ebi.ac.uk/pdbe/emdb/) under accession codes: EMD-2031 (FLNa16-24, unsymmetrized), EMD-2032 (FLNa16-24 symmetrized), EMD-2033 (FLNa16-24 + $\beta$ peptide, unsymmetrized) and EMD-2034 (FLNa16-24 $+\beta$ peptide, symmetrized)

1 Present address: Department of Biochemistry, University of Oulu, Oulu, Finland.

2 Correspondence may be addressed to either of these authors (email ulla.m.pentikainen@jyu.fi or jylanne@jyu.fi). 
A

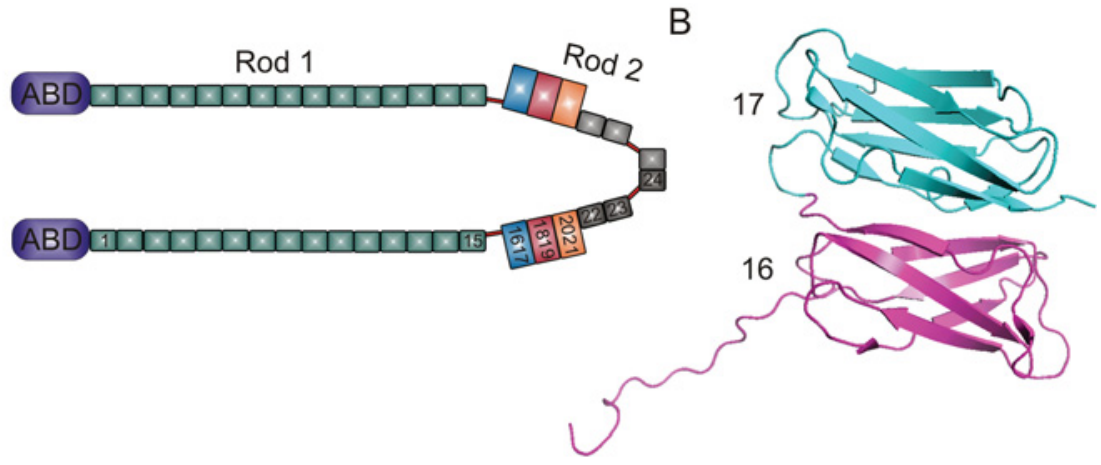

C
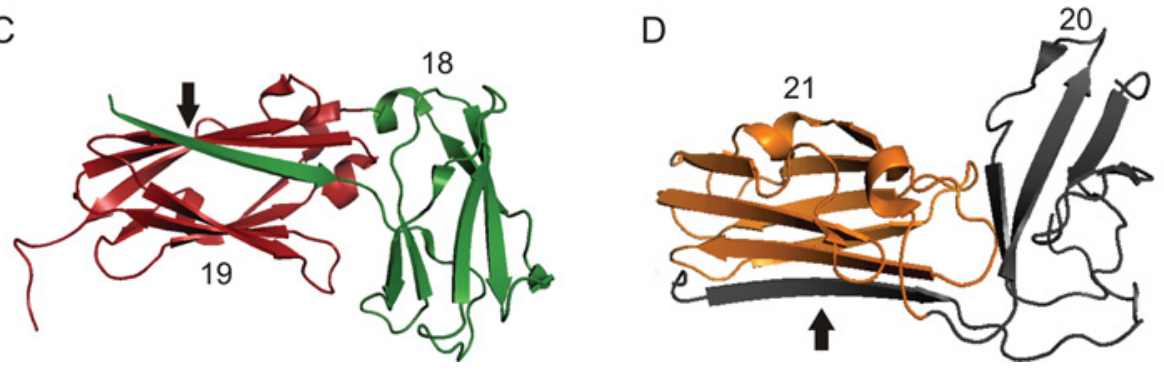

Figure 1 Location and known structures of the domains studied

(A) A schematic representation of human FLNa. The N-terminal actin-binding domains (ABDs) are shown as purple, Ig-like domains in rod 1 as green and Ig-like domains $22-24$ in rod 2 as grey. Domain pairs in rod 2 are presented as bigger boxes as blue (16-17), red (18-19) or orange (20-21). The C-terminal domains 24 are the dimerization domains. (B) The solution structure of human FLNa domain pair 16-17 (PDB code 2K7P) is presented as cartoon. Domain 16 is shown as pink and 17 as cyan. (C) The solution structure of human FLNa18-19 (PDB code 2K7Q) domain pair is shown as cartoon. Domains are displayed as green (18) or red (19). The arrow points to the A-strand of FLNa18. (D) Domains 20 (grey) and 21 (orange) of the crystal structure of three domain fragment of human FLNa19-21 (PDB code 2J3S) are shown as cartoon. The arrow points to the A-strand of FLNa20.

in D. melanogaster. Loss of binding to one or more of the numerous FLN-binding partners is a probable cause of many of the observed phenotypes, but the molecular mechanism of most of these mutations remains to be elucidated [24].

In the present study we show a low-resolution structure of the nine C-terminal domains of FLNa, based on a combinatorial use of EM (electron microscopy) and SAXS (small-angle X-ray scattering) techniques. This and a recent study using NMR techniques [25] show that the three domain pairs make a compact, but flexible, three-branched structure. The structural model, together with peptide interaction and mutagenesis studies, suggests that the domain pairs act independently of each other, thus facilitating large conformational changes in the fragment.

\section{EXPERIMENTAL}

\section{Recombinant proteins}

FLNa (UniProt entry P21333) fragments were amplified by PCR and cloned into a modified pGEX vector (GE Healthcare) with a TEV (tobacco etch virus) protease cleavage site. The domain boundaries were based on the published alignment [5]. GST (glutathione transferase) fusion proteins were expressed in Escherichia coli strain BL21 GOLD and purified as described previously [14]. The L1963E and I2144E mutations were introduced using the QuikChange ${ }^{\circledR}$ protocol (Stratagene).

\section{SAXS (small-angle X-ray scattering)}

SAXS data were collected at the EMBL beamline X33 at the DORIS III storage ring, DESY (Hamburg, Germany) [26]. The measurements were carried out at $288 \mathrm{~K}$ in $20 \mathrm{mM}$ Tris
(pH 8.0), $100 \mathrm{mM} \mathrm{NaCl}$ and $10 \mathrm{mM}$ DTT (dithiothreitol). The concentrations of FLNa fragments were adjusted to $3-6 \mathrm{mg} / \mathrm{ml}$ and FLNa fragments and peptides were used at a molar ratio of 1:10. MAR345 image plate at sample-detector distance $2.7 \mathrm{~m}$ and wavelength $\lambda=0.15 \mathrm{~nm}$, covering the momentum transfer range $0.08<\mathrm{s}<4.9 \mathrm{~nm}^{-1}[\mathrm{~s}=4 \pi \sin (\theta) / \lambda$, where $2 \theta$ is the scattering angle] was used. The data were processed using standard procedures by the program package PRIMUS [27]. $R_{\mathrm{g}}$ and $D_{\max }$ values were evaluated using the program GNOM [28].

The $a b$ initio envelopes were obtained by averaging 10-20 independent runs from the bead modelling program GASBOR [29] with the program DAMAVER [30]. The rigid-body modelling of FLNa16-21 was performed with the program SASREF [31] using FLNa domain pairs 16-17 (PDB code 2K7P), 18-19 (PDB code $2 \mathrm{~K} 7 \mathrm{Q}$ ) and 20-21 (PDB code $2 \mathrm{~J} 3 \mathrm{~S}$ ) as rigid bodies. The rigid-body models were fitted into the $a b$ initio envelopes using the SITUS program package [32] (http://situs.biomachina.org/). The images of the SAXS models were made with the program VMD [33] (http://www.ks.uiuc.edu/Research/vmd/) or PyMOL (http://www.pymol.org).

\section{Binding assays}

The synthetic peptides of migfilin (residues 5-19), $\beta 2$ cytoplasmic tail (residues 745-765) and DRD3 (residues 210230) (EZBiolab) were coupled to NHS ( $N$-hydroxysuccinimide)activated Sepharose 4 Fast Flow (GE Healthcare) at 277 K according to the manufacturer's instructions. Purified FLNa fragments were incubated for $1 \mathrm{~h}$ at $296 \mathrm{~K}$ with $15 \mu \mathrm{l}$ of the peptide-Sepharose in $1 \%$ Triton X-100, $150 \mathrm{mM} \mathrm{NaCl}$ and $20 \mathrm{mM}$ Tris (pH 7.4). The Sepharose was centrifuged at $2000 \mathrm{~g}$ for 2 min and washed three times with $300 \mu$ l of the binding buffer. 
Proteins were then eluted with $10 \mu \mathrm{l}$ of SDS electrophoresis sample buffer and run on a SDS/PAGE (12\% gel). The intensities of Coomassie Blue-stained protein bands were then evaluated by ImageJ (http://rsbweb.nih.gov/ij/). The data were plotted as the percentage of maximal binding and fitted to equation $\mathrm{Y}=$ $B_{\max } * \mathrm{X} /\left(K_{\mathrm{d}}+\mathrm{X}\right)$ using GraphPad Prism 5 (GraphPad Software).

\section{EM}

For cryo-EM, $2 \mu \mathrm{l}$ of sample was placed on a glow-discharged lacey carbon film EM grid, blotted and plunge-frozen in liquid ethane. The samples were imaged on an FEI F30 electron microscope operating at $300 \mathrm{kV}$ and with a magnification of $\times 59000$. Micrographs without evidence of astigmatism, drift or excessive defocus were digitized using a Z/I Photoscan scanner giving a pixel size at the specimen of $1.1865 \AA(1 \AA=0.1 \mathrm{~nm})$, interpolated for processing to $2.37 \AA$. The defocus was determined and the micrographs further triaged using ctffind 3 . The particles were picked using the EMAN program Boxer [34], after contrast enhancement in MATLAB via a self-written routine in which a three-level decomposition with a fourth-order bi-orthogonal wavelet function was applied to the images. The usefulness of this particular filter was determined empirically and on the basis of the positions so identified for the FLN molecules. Unfiltered particles were extracted, again in Boxer, and then corrected for their CTFs (contrast transfer functions) using the EMAN program applyctf [34]. Following this, the images of FLNa16-24 were subjected to multivariate statistical analysis in IMAGIC [35] to determine class averages (e.g. Supplementary Figures S3A and S3B at http://www.BiochemJ.org/bj/446/bj4460261add.htm). The number of eigenimages used in classification was determined empirically and then resulting class sums with well-defined molecular density and low background signal were used in angular reconstitution to determine models, initially assuming no symmetry. The resulting unsymmetrized maps were then refined in SPIDER [36] operated within a self-written graphical user interface for iterative refinement of structures (J.F. Flanagan IV and R.J.C. Gilbert, unpublished work). An angular spacing of $15^{\circ}$ was used throughout. Independent maps of FLN and FLN with peptide both showed an apparent 2fold axis of symmetry and, to demonstrate this, self-rotation functions were used employing CNS [37] and using information between $200 \AA$ and $30 \AA$ resolution, displayed using Gropat (R.M. Esnouf, unpublished work) (Supplementary Figure S4A at http://www.BiochemJ.org/bj/446/bj4460261add.htm). Because the self-rotation functions showed both a 2 -fold $\left(\kappa=180^{\circ}\right)$ and a 2 -fold distribution of 3 -fold $\left(\kappa=120^{\circ}\right)$ axes and because the class averages and eigenimages demonstrated unequivocal dyad axes of symmetry apparent as pseudo-mirror planes (Supplementary Figures S3A and S3C) and the maps themselves appeared to have dyad axes of symmetry (Figure 4A), we then repeated the reconstruction process from the start using $\mathrm{C} 2$ symmetry, but otherwise as above. The angular distributions determined for the particles (Supplementary Figure S4B) were reasonably even; for FLN the mean number of particles per bin was 252, the median 215, the minimum 116 and the maximum 404. For FLN with peptide the mean was 179 , the median 167 , the minimum 124 and the maximum 300. Throughout, resolution was assessed by FSC (Fourier shell correlation) with a 0.5 FSC cut-off. WellMAP programs (J.F. Flanagan IV and R.J.C. Gilbert, unpublished work) were used to generate the FSC and particle distribution plots for the data. The Fourier shell correlations showed sharp resolution cut offs and low residual noise (Supplementary Figure $\mathrm{S} 4 \mathrm{C})$, but indicated resolutions of $15.6 \AA$ and $15.7 \AA$ for the unsymmetrized reconstructions of FLNa16-24 without and with $\beta 7$ peptide respectively, and resolutions of $12.2 \AA$ and $12.8 \AA$ for the corresponding symmetrized maps. Alignment to a common reference can result in spuriously high resolution estimations due to the correlation of noise [38] and a recent paper has shown angular assignment errors of $\sim 10^{\circ}$ in particles twice the size of the FLNa16-24 dimer [39]. The distinctive two-pronged shape of the FLNa rod 2 segment may have assisted in increasing the accuracy of alignment, as also the symmetry may have done, which is a powerful feature in the classification (Supplementary Figure S3); Henderson and colleagues have pointed out how more accurate alignment may be assisted by distinctively shaped projected objects [39]. The self-rotation functions are nevertheless a striking validation of the reconstructions as they clearly do show unimposed symmetry being detected in the maps (Supplementary Figure S4A). By restricting the alignment to the inherently coarse $15^{\circ}$, we have avoided over-determination of Euler angular values and the maps were ultimately filtered to $18 \AA$ because of this limited sampling in projection determination, whereas finer samplings resulted in the accumulation of noise. The unsymmetrized maps were correspondingly filtered to $30 \AA$ A. Filtration was performed using the program WellMAP (J.F. Flanagan IV and R.J.C. Gilbert, unpublished work) in which a Gaussian filter was applied to the data. WellMAP programs were also used to generate the FSC and particle distribution plots for the data. EM maps were displayed and fitting to them was performed using Chimera software [40].

\section{Structure submission}

The cryo-EM reconstructions reported in this manuscript have been deposited in the European Bioinformatics Institute Electron Microscopy Database (http://www.ebi.ac.uk/ pdbe/emdb/) under accession codes: EMD-2031 (FLNa16-24, unsymmetrized), EMD-2032 (FLNa16-24 symmetrized), EMD2033 (FLNa16-24 + $\beta$ peptide, unsymmetrized) and EMD-2034 (FLNa16-24 $+\beta$ peptide, symmetrized).

\section{RESULTS}

\section{SAXS analysis of a six domain fragment of FLNa}

To study the structure of the rod 2 region of FLNa, a six domain fragment, FLNa16-21, was analysed by SAXS. The shapes of the scattering profile and distance distribution functions (Figure 2A) indicated that the fragment has a complex architecture, where domains are neither linearly nor globularly organized, but rather have a branched arrangement. The radius of gyration $\left(R_{\mathrm{g}}\right)=3.42$ $( \pm 0.01) \mathrm{nm}$ and the maximum linear dimension $\left(D_{\max }\right)=12$ $( \pm 1) \mathrm{nm}$ (Table 1), suggest a compact organization of domains in the fragment, consistent with results for individual domain pairs that were earlier measured to be $R_{\mathrm{g}}=1.9-2.1 \mathrm{~nm}$ and $D_{\max }=$ $6.0-6.5 \mathrm{~nm}$ [14]. A total of ten independent ab initio models were calculated. They fitted to the experimental data with a discrepancy $(\varphi)$ of 1.1-1.7; the mean normalized spatial discrepancy between the models was 1.30 , showing that the models agree well with each other. The average of the most populated $a b$ initio envelope demonstrates a planar, three-branched shape which consists of three equally long $(\sim 5.5 \mathrm{~nm})$ arms (Figure $2 \mathrm{~B})$.

The SAXS data was also used to fit the atomic structures of the three domain pairs within FLNa16-21 [13,14], by rigidbody modelling. The resulting model gave an excellent fit to the experimental scattering data $(\chi=0.91$; Figure $2 \mathrm{~A})$ and suggested that domain pairs are organized in the three-branched shape so 


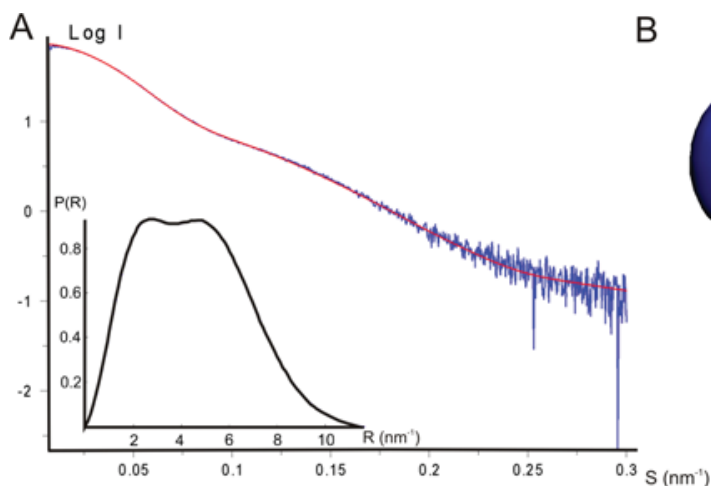

C

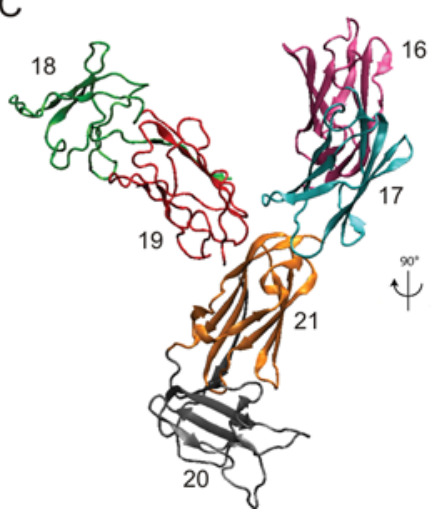

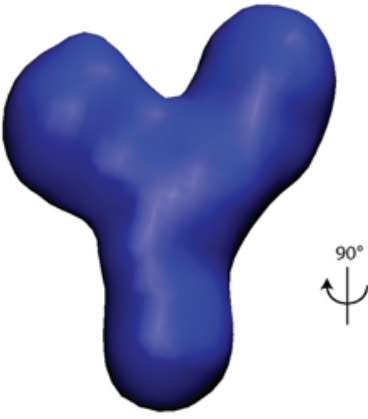

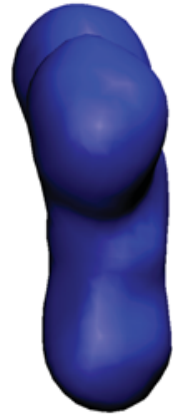

$\mathrm{D}$

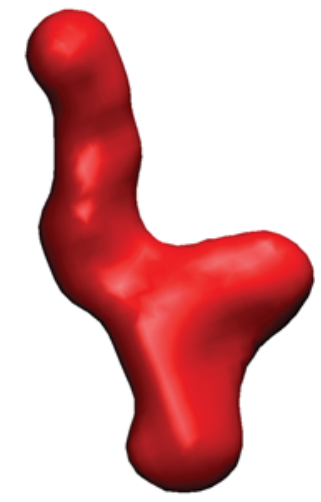

Figure 2 SAXS models of FLNa16-21 revealed three-branched shape

(A) Experimental scattering pattern of FLNa16-21 in plotted in blue and the fit obtained by SASREF corresponding to the model in (C) is presented as red solid line. The plot displays the logarithm of the scattering intensity as a function of momentum transfer $\mathrm{s}\left(\mathrm{nm}^{-1}\right)$. The distance distribution function of FLNa16-21 computed by GNOM is shown in the insert. Two peaks in the distance distribution curve are indicative of bimodal distance distribution. (B) Averaged ab initio envelope of FLNa16-21 obtained by GASBOR and averaged by DAMAVER is displayed in two orthogonal views. (C) The rigid-body model of FLNa16-21 based on the experimental scattering data is presented as cartoon. Every FLNa domain is shown as different colour; 16 as pink, 17 as cyan, 18 as green, 19 as red, 20 as grey and 21 as orange. The model is shown in three orthogonal views. The last view displays the model fitted into the ab initio envelope shown in (B). (D) The ab initio envelope of FLNa15-21 verifies the location of the N-terminus in FLNa16-21 models (not to the same scale as $\mathbf{B}$ and $\mathbf{C}$ ).

Table 1 SAXS parameters of FLNa fragments with and without peptides

\begin{tabular}{llc}
\hline Sample & $R_{g}(\mathrm{~nm})$ & $D_{\max }(\mathrm{nm})$ \\
\hline FLN1621 & $3.42 \pm 0.01$ & 12 \\
FLNa1521 & $4.07 \pm 0.01$ & 15 \\
FLNa1621 + integrin $\beta 2$ & $3.47 \pm 0.01$ & 12 \\
FLNa1621 + migfilin & $4.08 \pm 0.02$ & 14 \\
FLNa1621 + migfilinl15E & $3.40 \pm 0.01$ & 11 \\
FLNa1621 + DRD3 & $3.90 \pm 0.01$ & 15 \\
FLNa1621 + DRD3 + migfilin & $4.21 \pm 0.01$ & 15 \\
FLNa162112144E & $3.60 \pm 0.01$ & 11 \\
FLNa162112144E + integrin $\beta 2$ & $3.67 \pm 0.01$ & 13 \\
FLNa162112144E + migfilin & $3.95 \pm 0.02$ & 13 \\
FLNa1621L1956E & $3.60 \pm 0.02$ & 12 \\
FLNa1621L1956E + DRD3 & $4.03 \pm 0.01$ & 13 \\
FLNa1621L1956EI2144E & $3.65 \pm 0.01$ & 12 \\
FLNa1621L1956EI2144E + DRD3 + migfilin & $5.11 \pm 0.02$ & 17 \\
& & \\
\hline
\end{tabular}

that each arm consists of one domain pair (Figure 2C). The odd-numbered domains are close to the centre of the structure and the even domains are outermost. Apart from the polypeptide connection between the domains, the model does not indicate any other stabilizing interactions between domains, consistent with the NMR observations [25,41]. Superposition of the $a b$ initio and rigid-body models reveals excellent convergence of the two independent approaches (Figure 2C, right-hand panel).
To define the position of the N-terminus in FLNa1621, the scattering data for the FLNa fragment 15-21 were recorded (Supplementary Figure S1 at http://www.BiochemJ.org/ bj/446/bj4460261add.htm). The $a b$ initio shape of FLNa15-21 clearly shows that one dimension is longer than the other two dimensions (Figure 2D), unlike the shape of FLNa16-21 where all of the arms are equally long. Accordingly, it seems likely that domain 16 , separated from domain 15 by a 32 amino acid linker [5], is located at the tip of one arm of the FLNa16-21 envelope (Figure 2B), consistent with the rigid-body model (Figure 2C).

\section{Integrin, migfilin and DRD3 peptides induce conformational flexibility in FLNa}

The SAXS data presented above and a recent NMR study [25] show that the individual domain pairs in the rod 2 region of FLNa interact very little with each other, and that the overall structure is rather flexible [25]. To further study the flexibility of the fragment, we tested whether the FLN-binding transmembrane receptor cytoplasmic domain or signalling adaptor protein peptides can cause conformational changes in the FLNa16-21 fragment. The conformational changes were initially analysed by SAXS. The peptides used were so small that their direct scattering could be subtracted from the data to reveal their effect on the FLN fragment structure. To our surprise, millimolar concentrations of migfilin and DRD3 peptides increased the $R_{\mathrm{g}}$ value of FLNa1621 by $0.48-0.66 \mathrm{~nm}$ and the $D_{\max }$ value by $2-4 \mathrm{~nm}$ (Table 1 ). 
A

binding to migfilin

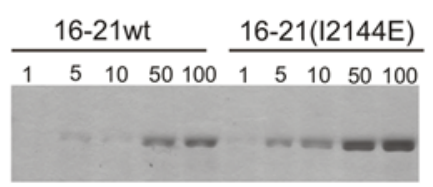

binding to DRD3

16-21wt

1 control

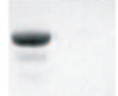

C

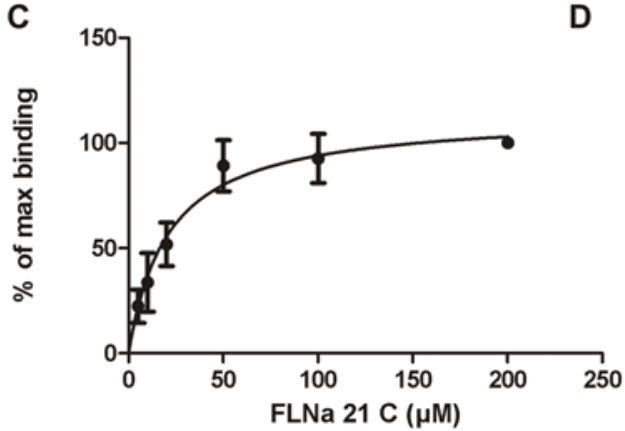

B
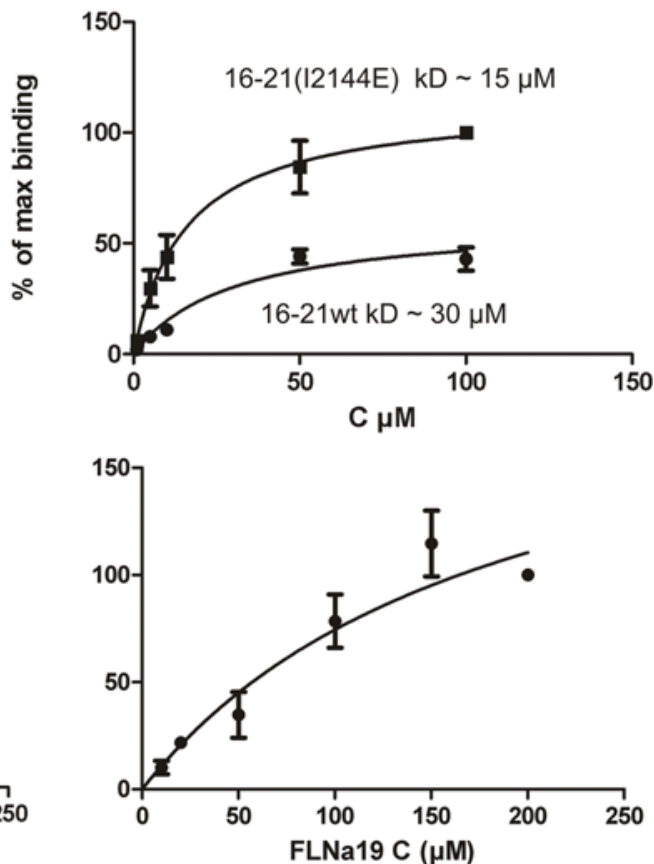

E

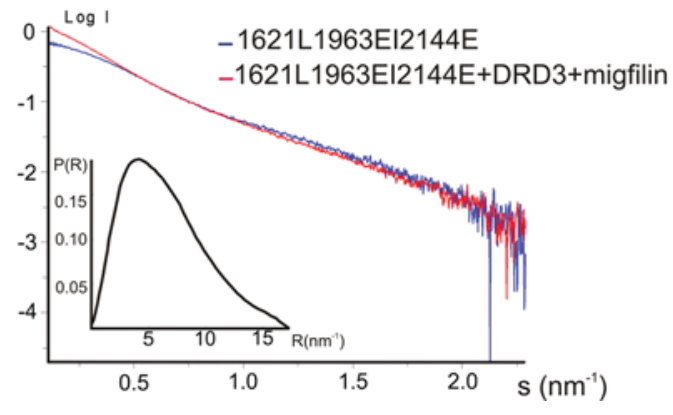

Figure 3 Analysis of filamin mutants

(A) FLNa16-21wt and FLNa16-21 I2144E mutant binding to migflin peptide in protein concentrations of 1, 5, 10, 50 and $100 \mu \mathrm{M}$ assayed by protein staining of pull-down assays and FLNa16-21wt binding to DRD3 in the protein concentration of $1 \mu \mathrm{M}$.(B) FLNa16-21wt and FLNa16-21 I2144E mutant binding to migfilin was quantified by densitometry and expressed as the percentage of maximum filamin fragment binding in each experiment. (C) FLNa21 and (D) FLNa19 binding curve to migfilin. (E) FLNa16-21wt binding to DRD3 in the protein concentration of $1 \mu$ M. (F) The scattering curve of FLNa16-21L1963EI2144E alone is blue and FLNa16-21L1963E I2144E with migfilin and DRD3 peptides is red. Distance distribution function of FLNa16-21L1963E I2144E + DRD3 + migfilin is shown in the insert. wt, wild-type.

Scattering and distance distribution curves from each experiment are presented in Supplementary Figure S2 at http://www.BiochemJ.org/bj/446/bj4460261add.htm. A nonbinding migfilin mutant (I15E) peptide [17] did not induce any changes in the $R_{\mathrm{g}}$ and $D_{\max }$ values (Table 1), validating the method and suggesting that the peptide binds to the FLNa16-21 in a similar way to its binding to domain 21 alone [17].

We next wanted to further verify the idea that ligand peptides bind to the $\mathrm{CD}$ faces of domains 19 and 21, displace the A-strands of the preceding domains and thus cause global conformation changes in the FLNa rod 2. To do this, interaction destabilizing mutations were introduced in the A stands that mask the binding sites (L1956E and I2144E). The I2144E mutation enhanced the migfilin binding to FLNa16-21 to similar levels as to FLNa21 alone (Figure 3A-3C). In the absence of ligand peptides, these mutations did not cause significant changes to the molecular shape parameters of FLNa16-21 in SAXS (Table 1), but in the presence of peptides both the $R_{\mathrm{g}}$ and $D_{\max }$ values were observed to increase (Table 1). The previously characterized peptide from the cytoplasmic tail of integrin $\beta 2$, which has lower affinity than the $\beta 7$ peptide [16] induced changes in FLNa16-21 conformation only when the FLNa20 A-strand interaction with FLNa21 CD face was weakened by the I2144E mutation (Table 1). Although all of the interacting peptides changed the scattering patterns (Supplementary Figure S2), the most significant changes in the scattering profile and distance distribution function of FLNa1621 were seen when both migfilin and DRD3 peptides and both FLNa18 and 20 A-strand mutations (L1956E and I2144E) were introduced at the same time (Figure 3E). In the presence of the peptides, the $R_{\mathrm{g}}$ value increased to $5.11 \mathrm{~nm}$ and the $D_{\max }$ value up to $17 \mathrm{~nm}$ (Table 1), suggesting a significantly more elongated shape for FLNa16-21. Also the bimodal distance distribution seen without peptides or with a single peptide (Figure 2 and Supplementary Figure S2) changed to a single major distance peak when two mutations and two peptides were present simultaneously (Figure 3E, insert). These findings indicate major 
A
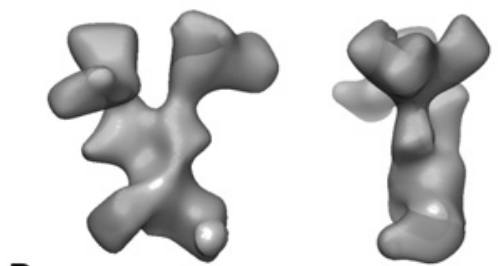

B

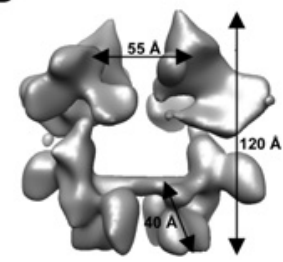

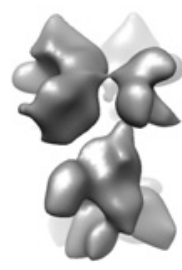
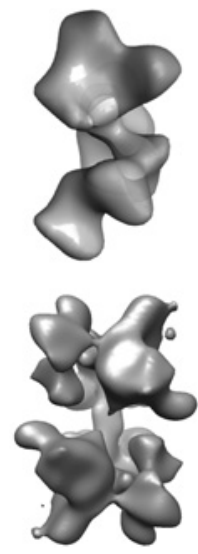

Figure 4 Cryo-EM reconstructions of FLNa16-24

(A) The initial reconstruction was generated without imposed symmetry. This map was filtered to $30 \AA$. (B) The final reconstruction was generated by imposing 2-fold symmetry and filtered to $18 \AA$. In both cases three orthogonal views are shown. The overall dimensions of the final model are marked.

flexibility in the FLNa16-21 fragment and are consistent with the prediction that DRD3, integrin and migfilin peptide binding detaches the A-strands of domains 18 and 20 from the CD faces of domains 19 and 21. Furthermore, the results support the idea that the 18-19 and 20-21 domain pairs can interact and change conformation independently of each other.

\section{Single particle EM reconstruction of the C-terminal part of FLNa}

To gain independent structural data of the FLNa rod 2 region, single particle cryo-EM was used to calculate three-dimensional reconstructions of a nine domain fragment (FLNa16-24) that forms a $185 \mathrm{kDa}$ homodimer. A total of 21133 particles were classified and the reconstructions were initially generated with no symmetry assumed (see the Experimental section). The reconstruction showed features of 2-fold symmetry (Figure 4A) that could also be seen the image class-averages (Supplementary Figure S3A) and in a self-rotation analysis of the reconstruction (Supplementary Figure S4A). Two 3-fold symmetry peaks were also seen in the corresponding self-rotation function plot (Supplementary Figure S4A). The global 2-fold symmetry could be used to improve the overall quality of the reconstruction by reprocessing the original images assuming 2-fold symmetry. Figure 4(B) shows the resulting reconstruction of FLNa16-24 in orthogonal views. The nominal resolution of the reconstruction was $12.2 \AA$ (Supplementary Figure S4C), but we filtered the maps to $18 \AA$ for display purposes because the sampling angle was $15^{\circ}$. The particle orientation distribution was relatively even and all views were represented (see the Experimental and Supplementary Figure S4B, left-hand panel). The symmetrized FLNa16-24 map (Figure 4B) has a distinctive two-pronged appearance similar to that seen in the unsymmetrized map (Figure 4A). The dimerization interface is found towards the base of the fork, at the upper edge of a pair of globular lobes of density. Above this, the structure displays a central stem for each prong, which bifurcates into a smaller and a larger piece of density resulting in a threebranched structure. The connections between all of the lobes are not contoured at the density level chosen for the isosurface representation: this may be a result of limited resolution, limited flexibility in linker regions or a combination of such factors.

FLNa16-24 has overall dimensions of $120 \AA \times 120 \AA \times 60 \AA$; the distance between the prongs is $55 \AA$ and the distance from the base of the molecule to the point of bifurcation is $40 \AA$.

To assist in the interpretation of the FLNa16-24 EM reconstruction, we fitted $a b$ initio SAXS envelopes in it. A fit of the FLNc23-24 ab initio SAXS envelope [9] was poor (Supplementary Figure S4D), but the FLNa16-21 envelope fitted well into the forked density of the EM map (correlation coefficient 0.68), leaving an appropriate space for FLNa22-23 [14] (correlation coefficient 0.90) (Figure 5A). The connection of domain 22 to the centre of the three-branched structure is consistent with the polypeptide connections between the $\mathrm{C}$ terminus of domain 21 and the N-terminus of domain 22. Thus, with the help of SAXS data, we are able tentatively to assign eight Ig-like domains in the EM reconstruction.

To explore the ligand peptide-induced conformation changes in FLNa rod 2 region, we performed single particle EM analysis of FLNa16-24 in the presence of integrin $\beta 7$ peptide. A cryo-EM image set of 32477 particles for FLNa16-24 with integrin $\beta 7$ peptide was analysed independently. Representative class averages are shown in Supplementary Figure S3(B). The final map was generated with 2-fold symmetry constrains. Resolution estimation and particle orientation distribution for this reconstruction are shown in Supplementary Figures S4(B) and S4(C). Both the unsymmetrized (Supplementary Figure S4E) and symmetrized (Figure $5 \mathrm{~B}$ ) reconstructions showed a more open conformation of the FLNa16-24 fragment with the integrin peptide than without it.

\section{DISCUSSION}

In the present study we have investigated the overall structure of the C-terminal rod 2 fragment of FLNa that comprises nine Ig-like domains (domains 16-24). This fragment is of considerable interest in relation to FLN function, first, because it mediates FLN dimerization that is essential for actin crosslinking and, secondly, because it contains several interaction sites for transmembrane and signalling proteins. Our current results indicate that the rod 2 fragment is compact, but flexible, and that the flexibility responds to and contributes to the regulation of protein interactions.

Rotary shadowing EM studies have previously revealed that rod 2 of FLN is more tightly packed than the first 15 Ig domains comprising rod 1 [12]. Our current data show that the first six domains of rod 2 form a three-branched, rather planar structure, where each previously characterized domains pair (16-17, 1819 and 20-21) forms one branch. Our SAXS results confirm the recent NMR-based model from the same six domain fragment [25]. Overall the longest diameter of the nine domain rod 2 fragment is about $15 \mathrm{~nm}$. This diameter fits with the estimation of $19 \pm 6 \mathrm{~nm}$ based on rotary shadowing EM [12].

The electron microscopy reconstructions are consistent with a single contact site between the two arms of the structure. This supports previous results that dimerization of vertebrate FLNs is mediated only by the last, the 24th, Ig-like domain $[9,11,42,43]$. However, we were not able to exactly position FLNa24 in our EM reconstruction. In the map, the dimerization connection appeared as a narrow bridge that is inconsistent with the known structure of the FLN dimerization interface. The crystal structures show that the 24th Ig-like domain of FLNc and FLNa form a long interface where one of the $\beta$-sheets continues from one monomer to another $[11,43]$. This was not seen in our EM map, although we were able to fit the SAXS envelope of FLNa22-23. We suspect that the reasons for this are the limited resolution of the reconstruction, 

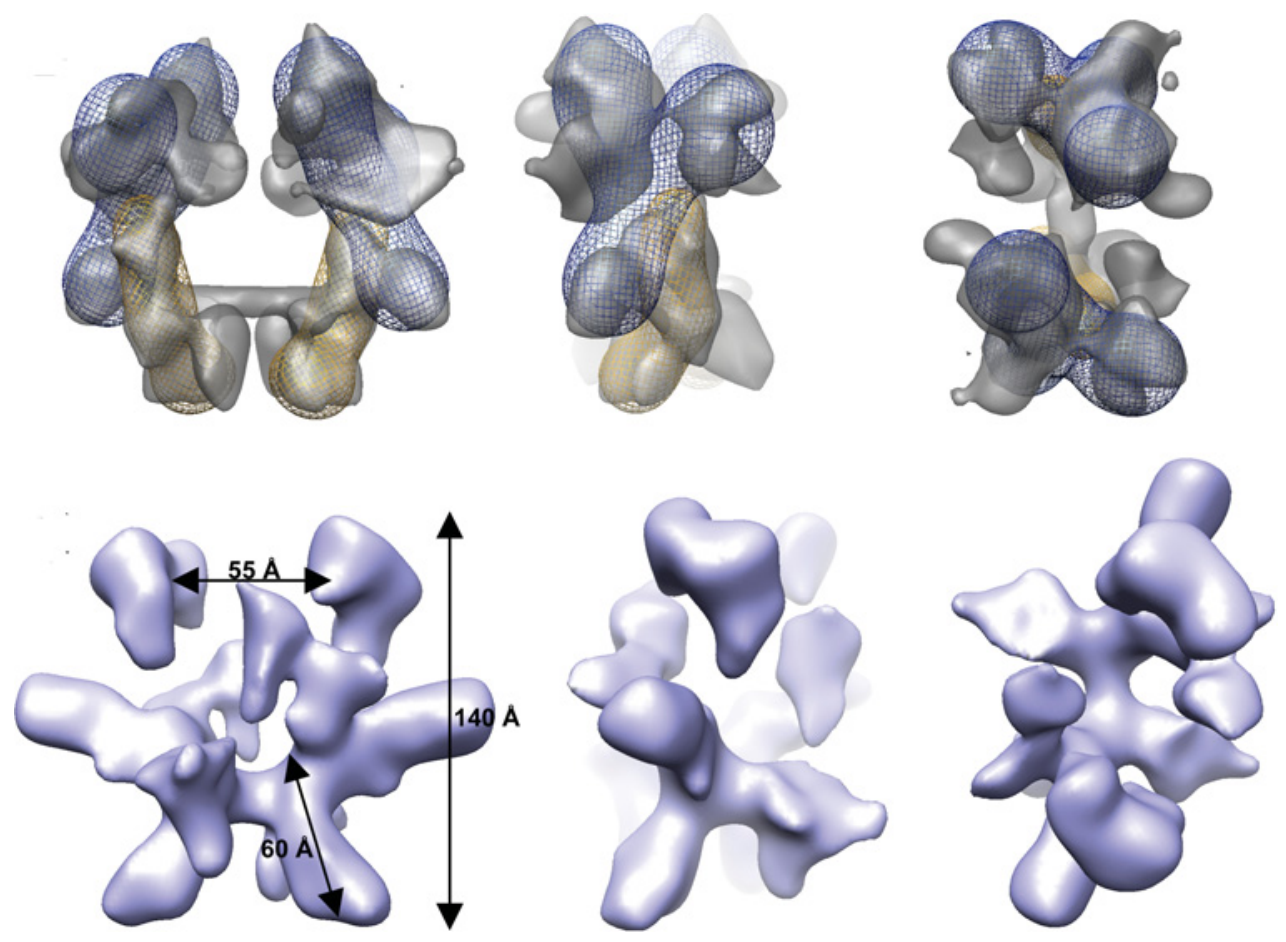

Figure 5 Cryo-EM reconstruction of FLNa16-24: analysis and effect of integrin peptide

(A) Fit of the SAXS ab initio envelopes for FLNa16-21 (blue) and FLNa22-23 (yellow) to the cryo-EM map shown in Figure 4. (B) Cryo-EM reconstruction of FLNa16-24 with the $\beta_{7}$ peptide bound Dimensions are marked as in Figure 4.

the use of 2-fold symmetry and the flexibility of the hinge 2 region between domains 23 and 24. Earlier SAXS analysis has shown that there are few contacts between FLNc domains 23 and 24 , which may allow flexibility of the hinge 2 region [9]. Thus our map might represent an average of multiple conformations of domain 24 in relation to the rest of the molecule.

The three-branched shape of the six-domain fragment, FLNa16-21, was revealed by ab initio analysis of the SAXS data. The same shape was independently reconstituted by fitting known high-resolution two-domain fragments to the SAXS scattering. This shape fits very well with the fact that, in the previously determined atomic structures the $\mathrm{N}$ - and C-termini of two domains, pairs locate close to each other $[13,14]$. The position of the N-terminus of the six-domain fragment could be verified by SAXS analysis of an N-terminally extended fragment. The EM reconstruction provided support for the shape as well and for the position of the C-terminus of the fragment. Given the limitations of the resolution of the SAXS measurements, we believe that we have been able to generate a reliable model for the organization of the six domains. What are the implications of the model for FLN function? First, the model shows that there are only a very limited number of interaction sites between the three domain pairs. This implies that the structure has intrinsic flexibility, the domain pairs can wobble in relation to each other. Flexibility is supported by the finding that the angle between domains 19 and 21 is quite different in this SAXS envelope than in the crystal structure of the three-domain fragment FLNa19-21 [13]. Molecular dynamics simulations suggest that the observed angle in the crystal may be partly due to crystal packing [13]. Flexibility between individual domains and especially between the domain pairs is also supported by NMR analysis [25]. A second implication of the current model is that the binding sites in FLN domains 17, 19 and 21 are located quite far apart in space, and are thus expected to function independently. We earlier speculated that there could be cross-talk between different binding sites so that for instance protein binding to domain 19 could allosterically regulate the masking or unmasking of domain 21 , or vice versa [13]. The current model suggests that this might not be the case.

In addition to the inter-domain flexibility of the filamin Cterminal fragment, we have shown in the present study that transmembrane receptor and signalling molecule binding to the domain pairs 18-19 and 20-21 can induce large changes in the overall structure. The integrin $\beta 7$ peptide caused marked changes in the FLNa16-24 EM reconstruction. The migfilin and DRD3 peptides induced a clear elongation of the FLNa16-21 fragment, increasing the maximum distance by approximately $2 \mathrm{~nm}$. The failure of a non-FLN-binding migfilin peptide to induce such a conformational change and the responsiveness of the changes to FLN mutations that weaken the masking of CD faces of domains 19 and 21, are consistent with a model where the peptides displace A-strands of domains 18 and 20 from the CD face of domains 19 and 20 respectively. The most elongated shape of FLNa16-21, that has a maximum distance of $17 \mathrm{~nm}$ was achieved when both of the A-strand mutations and both the DRD3 and migfilin peptides were introduced. In this elongated shape, both of the A-strands have presumably been detached, whereas migfilin alone displaces only the A-strand of FLNa20. The requirement of both binding events for maximal elongation is consistent with the idea that the two binding sites act independently. A model for the peptide-induced conformational changes is presented in Figure 6. We and others have also observed similar conformational changes in shorter FLN fragments [44,45]. It has been recently reported that the rod 2 region of FLN can undergo conformational changes under low $(<10 \mathrm{pN})$ forces, whereas changes in the rod 1 region are mainly observed at forces more than $30 \mathrm{pN}$, which may lead to domain unfolding [46]. Our branched and compact structure of the 


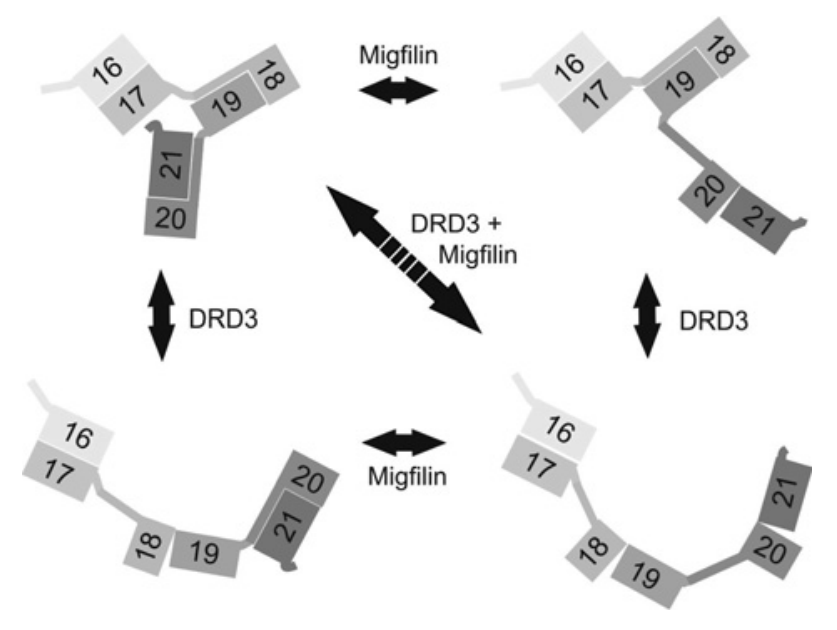

Figure 6 Schematic representation of domain arrangement of FLNa16-21 fragment and the conformational changes induced by DRD3 and migfilin peptide

rod 2 region suggests that there are very few contacts between the domain pairs and thus external forces can directly be channelled to open the masked binding sites in the domain pairs 18-19 and 20-21. These sites are in such an orientation with respect to the location of peptide connections [47] that would allow relatively weak forces to cause a zipper-like opening of binding sites, as observed in steered molecular dynamics simulations $[22,48]$. Although the force and time scales used in molecular dynamics simulations are not directly comparable with those in cells and in single-molecule experiments, the branched structure shown in the present paper for the rod 2 region can potentially explain how opening of domain pairs 18-19 and 20-21 could be the source of the extension observed at $<10 \mathrm{pN}$ forces [46], and how the strain in the FLN-actin network could enhance integrin binding to the network [23].

In summary, we have shown in the present study a complete, albeit low resolution, picture of the domain organization in the rod 2 segment of FLNa. This segment contains the dimerization site (domain 24), two canonical Ig domains (domains 22-23), and three intertwined domain pairs (16-17, 18-19 and 2021). Our model shows that there is considerable flexibility between these structural units. Furthermore, we show that transmembrane receptor and signalling protein binding induces large conformational changes that are consistent with partial opening of domain pairs 18-19 and 20-21. These results provide a basis for understanding the function of FLN as a cellular mechanosensor [23,49-52].

\section{AUTHOR CONTRIBUTION}

Salla Ruskamo, Gregor Hofmann, Robert Gilbert and Ulla Pentikäinen designed and performed the experiments and analysed the data. Pengju Jiang expressed and purified the dimeric protein. lain Campbell, Robert Gilbert and Jari Ylänne supervised and designed the experiments. All of the authors participated in the writing of the paper.

\section{ACKNOWLEDGEMENTS}

We thank Dr Peter Konarev and Dr Dmitri Svergun (EMBL/DESY, Hamburg, Germany) for help with SAXS data analysis, and EMBL/DESY Hamburg for the access to the beamline $X-33$. We thank Arja Mansikkaviita for excellent technical assistance, Dr David Calderwood and Dr Mohamed Bouaouina (Yale University School of Medicine, New Haven, CT, U.S.A.) for helping with binding assays, and Emilia Horttana for contributing to mutagenesis and biochemical assays.

\section{FUNDING}

This work was supported by the Academy of Finland [grant numbers 121393 (to U.P.), 114713 (to J.Y.) and 138327 (to J.Y.)], the National Doctoral Programme in Informational and Structural Biology (to S.R.), the BBSRC (Biotechnology and Biological Sciences Research Council) [grant number BB/F006845 (to P.J.)] and the NSFC (National Natural Science Foundation of China) [grant number 31100530 (to P.J.)]. The support of Instruct (http://www.instruct-fp7.eu/) is gratefully acknowledged for software license purchase.

\section{REFERENCES}

1 Evans, E. A. and Calderwood, D. A. (2007) Forces and bond dynamics in cell adhesion. Science 316, 1148-1153

2 Vogel, V. and Sheetz, M. (2006) Local force and geometry sensing regulate cell functions. Nat. Rev. Mol. Cell Biol. 7, 265-275

3 Feng, Y. and Walsh, C. A. (2004) The many faces of filamin: a versatile molecular scaffold for cell motility and signalling. Nat. Cell Biol. 6, 1034-1038

4 Stossel, T. P., Condeelis, J., Cooley, L., Hartwig, J. H., Noegel, A., Schleicher, M. and Shapiro, S. S. (2001) Filamins as integrators of cell mechanics and signalling. Nat. Rev. Mol. Cell Biol. 2, 138-145

5 van der Flier, A. and Sonnenberg, A. (2001) Structural and functional aspects of filamins. Biochim. Biophys. Acta 1538, 99-117

6 Popowicz, G. M., Schleicher, M., Noegel, A. A. and Holak, T. A. (2006) Filamins: promiscuous organizers of the cytoskeleton. Trends Biochem. Sci. 31, 411-419

7 Zhou, X., Tian, F., Sandzén, J., Cao, R., Flaberg, E., Szekely, L., Cao, Y., Ohlsson, C., Bergo, M. O., Borén, J. and Akyürek, L. M. (2007) Filamin B deficiency in mice results in skeletal malformations and impaired microvascular development. Proc. Natl. Acad. Sci. U.S.A. 104, 3919-3924

8 Zhou, A., Hartwig, J. H. and Akyürek, L. M. (2010) Filamins in cell signaling, transcription and organ development. Trends Cell Biol. 20, 113-123

9 Sjekloca, L., Pudas, R., Sjöblom, B., Konarev, P., Carugo, O., Rybin, V., Kiema, T. R., Svergun, D., Ylänne, J. and Djinovic Carugo, K. (2007) Crystal structure of human filamin C domain 23 and small angle scattering model for filamin C 23-24 dimer. J. Mol. Biol. 368, 1011-1023

10 Gorlin, J. B., Yamin, R., Egan, S., Stewart, M., Stossel, T. P., Kwiatkowski, D. J. and Hartwig, J. H. (1990) Human endothelial actin-binding protein (ABP-280, nonmuscle filamin): a molecular leaf spring. J. Cell Biol. 111, 1089-1105

11 Pudas, R., Kiema, T., Butler, P. J. G., Stewart, M. and Ylänne, J. (2005) Structural basis for vertebrate filamin dimerization. Structure 13,111-119

12 Nakamura, F., Osborn, T. M., Hartemink, C. A., Hartwig, J. H. and Stossel, T. P. (2007) Structural basis of filamin A functions. J. Cell Biol. 179, 1011-1025

13 Lad, Y., Kiema, T., Jiang, P., Pentikäinen, O. T., Coles, C. H., Campbell, I. D., Calderwood, D. A. and Ylänne, J. (2007) Structure of three tandem filamin domains reveals auto-inhibition of ligand binding. EMBO J. 26, 3993-4004

14 Heikkinen, 0. K., Ruskamo, S., Konarev, P. V., Svergun, D. I., livanainen, T., Heikkinen, S. M., Permi, P., Koskela, H., Kilpeläinen, I. and Ylänne, J. (2009) Atomic structures of two novel immunoglobulin-like domain pairs in the actin cross-linking protein filamin. J. Biol. Chem. 284, 25450-25458

15 Kiema, T., Lad, Y., Jiang, P., Oxley, C. L., Baldassarre, M., Wegener, K. L., Campbell, I. D., Ylänne, J. and Calderwood, D. A. (2006) The molecular basis of filamin binding to integrins and competition with talin. Mol. Cell 21, 337-347

16 Takala, H., Nurminen, E., Nurmi, S. M., Aatonen, M., Strandin, T., Takatalo, M., Kiema, T., Gahmberg, C. G., Ylänne, J. and Fagerholm, S. C. (2008) $\beta 2$ integrin phosphorylation on $\mathrm{Thr}^{758}$ acts as a molecular switch to regulate 14-3-3 and filamin binding. Blood 112, 1853-1862

17 Lad, Y., Jiang, P., Ruskamo, S., Harburger, D. S., Ylänne, J., Campbell, I. D. and Calderwood, D. A. (2008) Structural basis of the migfilin-filamin interaction and competition with integrin $\beta$ tails. J. Biol. Chem. 283, 35154-35163

18 Ithychanda, S. S., Das, M., Ma, Y., Ding, K., Wang, X., Gupta, S., Wu, C., Plow, E. F. and Qin, J. (2009) Migfilin, a molecular switch in regulation of integrin activation. J. Biol. Chem. 284, 4713-4722

19 Lin, R., Karpa, K., Kabbani, N., Goldman-Rakic, P. and Levenson, R. (2001) Dopamine D2 and D3 receptors are linked to the actin cytoskeleton via interaction with filamin A. Proc. Natl. Acad. Sci. U.S.A. 98, 5258-5263

20 Lin, R., Canfield, V. and Levenson, R. (2002) Dominant negative mutants of filamin A block cell surface expression of the D2 dopamine receptor. Pharmacology $\mathbf{6 6}, 173-181$

21 Pentikäinen, U. and Ylänne, J. (2009) The regulation mechanism for the auto-inhibition of binding of human filamin A to integrin. J. Mol. Biol. 393, 644-657 
22 Chen, H. S., Kolahi, K. S. and Mofrad, M. R. K. (2009) Phosphorylation facilitates the integrin binding of filamin under force. Biophys. J. 97, 3095-3104

23 Ehrlicher, A. J., Nakamura, F., Hartwig, J. H., Weitz, D. A. and Stossel, T. P. (2011) Mechanical strain in actin networks regulates FilGAP and integrin binding to filamin A. Nature 478, 260-263

24 Nakamura, F., Stossel, T. P. and Hartwig, J. H. (2011) The filamins: organizers of cell structure and function. Cell Adh. Migr. 5, 160-169

25 Tossavainen, H., Koskela, O., Jiang, P., Ylänne, J., Campbell, I. D., Kilpeläinen, I. and Permi, P. (2012) Model of a six immunoglobulin-like domain fragment of filamin a (16-21) built using residual dipolar couplings. J. Am. Chem. Soc. 134, 6660-6672

26 Roessle, M. W., Klaering, R., Ristau, U., Robrahn, B., Jahn, D., Gehrmann, T., Konarev, P., Round, A., Fiedler, S., Hermes, C. and Svergun, D. (2007) Upgrade of the small-angle $X$-ray scattering beamline X33 at the European Molecular Biology Laboratory, Hamburg. J. Appl. Cryst. 40, s190-s194

27 Konarev, P. V., Petoukhov, M. V., Volkov, V. V. and Svergun, D. I. (2006) ATSAS 2.1, a program package for small-angle scattering data analysis. J. Appl. Cryst. 39, $277-286$

28 Svergun, D. I. (1992) Determination of the regularization parameter in indirect-transform methods using perceptual criteria. J. Appl. Cryst. 25, 495-503

29 Svergun, D. I., Petoukhov, M. V. and Koch, M. H. (2001) Determination of domain structure of proteins from X-ray solution scattering. Biophys. J. 80, 2946-2953

30 Volkov, V. V. and Svergun, D. I. (2003) Uniqueness of ab initio shape determination in small-angle scattering. J. Appl. Cryst. 36, 860-864

31 Petoukhov, M. V. and Svergun, D. I. (2005) Global rigid body modeling of macromolecular complexes against small-angle scattering data. Biophys. J. 89, 1237-1250

32 Wriggers, W. (2010) Using Situs for the integration of multi-resolution structures. Biophys. Rev. 2, 21-27

33 Humphrey, W., Dalke, A. and Schulten, K. (1996) VMD: visual molecular dynamics. J. Mol. Graphics 14, 33-38

34 Ludtke, S. J., Baldwin, P. R. and Chiu, W. (1999) EMAN: semiautomated software for high-resolution single-particle reconstructions. J. Struct. Biol. 128, 82-97

35 van Heel, M., Harauz, G., Orlova, E. V., Schmidt, R. and Schatz, M. (1996) A new generation of the IMAGIC image processing system. J. Struct. Biol. 116, $17-24$

36 Shaikh, T. R., Gao, H., Baxter, W. T., Asturias, F. J., Boisset, N., Leith, A. and Frank, J. (2008) SPIDER image processing for single-particle reconstruction of biological macromolecules from electron micrographs. Nat. Protoc. 3, 1941-1974

37 Brunger, A. T., Adams, P. D., Clore, G. M., DeLano, W. L., Gros, P., Grosse-Kunstleve, R. W., Jiang, J. S., Kuszewski, J., Nilges, M., Pannu, N. S. et al. (1998) NMR system: a new software suite for macromolecular structure determination. 54, 905-921

Received 27 February 2012/18 May 2012; accepted 8 June 2012

Published as BJ Immediate Publication 8 June 2012, doi:10.1042/BJ20120361
38 Grigorieff, N. (2000) Resolution measurement in structures derived from single particles. Acta Crystallogr. D Biol. Crystallogr. 56, 1270-1277

39 Henderson, R., Chen, S., Chen, J. Z., Grigorieff, N., Passmore, L. A., Ciccarelli, L., Rubinstein, J. L., Crowther, R. A., Stewart, P. L. and Rosenthal, P. B. (2011) Tilt-pair analysis of images from a range of different specimens in single-particle electron cryomicroscopy. J. Mol. Biol. 413, 1028-1046

40 Pettersen, E. F., Goddard, T. D., Huang, C. C., Couch, G. S., Greenblatt, D. M., Meng, E. C. and Ferrin, T. E. (2004) UCSF Chimera: a visualization system for exploratory research and analysis. J. Comput. Chem. 25, 1605-1612

41 Mäntylahti, S., Koskela, O., Jiang, P. and Permi, P. (2010) MQ-HNCO-TROSY for the measurement of scalar and residual dipolar couplings in larger proteins: application to a 557-residue IgFLNa16-21. J. Biomol. NMR 47, 183-194

42 Himmel, M., Van Der Ven, P. F. M., Stöcklein, W. and Fürst, D. 0. (2003) The limits of promiscuity: isoform-specific dimerization of filamins. Biochemistry 42, 430-439

43 Seo, M. D., Seok, S. H., Im, H., Kwon, A. R., Lee, S. J., Kim, H. R., Cho, Y., Park, D. and Lee, B. J. (2009) Crystal structure of the dimerization domain of human filamin A. Proteins 75, 258-263

44 Ithychanda, S. S. and Qin, J. (2011) Evidence for multisite ligand binding and stretching of filamin by integrin and migfilin. Biochemistry $\mathbf{5 0}, 4229-4231$

45 Pentikäinen, U., Jiang, P., Takala, H., Ruskamo, S., Campbell, I. D. and Ylänne, J. (2011) Assembly of a filamin four-domain fragment and the influence of splicing variant- 1 on the structure. J. Biol. Chem. 286, 26921-26930

46 Chen, H., Zhu, X., Cong, P., Sheetz, M. P., Nakamura, F. and Yan, J. (2011) Differential mechanical stability of filamin A rod segments. Biophys. J. 101, 1231-1237

47 Gräter, F., Shen, J., Jiang, H., Gautel, M. and Grubmüller, H. (2005) Mechanically induced titin kinase activation studied by force-probe molecular dynamics simulations. Biophys. J. 88, 790-804

48 Pentikäinen, U. and Ylänne, J. (2009) The regulation mechanism for the auto-inhibition of binding of human filamin A to integrin. J. Mol. Biol. 393, 644-657

49 D'Addario, M., Arora, P. D., Fan, J., Ganss, B., Ellen, R. P. and McCulloch, C. A. (2001) Cytoprotection against mechanical forces delivered through $\beta 1$ integrins requires induction of filamin A. J. Biol. Chem. 276, 31969-31977

50 Kainulainen, T., Pender, A., D'Addario, M., Feng, Y., Lekic, P. and McCulloch, C. A. (2002) Cell death and mechanoprotection by filamin a in connective tissues after challenge by applied tensile forces. J. Biol. Chem. 277, 21998-22009

51 Gehler, S., Baldassarre, M., Lad, Y., Leight, J. L., Wozniak, M. A., Riching, K. M., Eliceiri, K. W., Weaver, V. M., Calderwood, D. A. and Keely, P. J. (2009) Filamin A- $\beta 1$ integrin complex tunes epithelial cell response to matrix tension. Mol. Biol. Cell 20, 3224-3238

52 Shifrin, Y., Arora, P. D., Ohta, Y., Calderwood, D. A. and McCulloch, C. A. (2009) The role of FilGAP-filamin A interactions in mechanoprotection. Mol. Biol. Cell 20, 1269-1279 


\section{SUPPLEMENTARY ONLINE DATA}

\section{The C-terminal rod 2 fragment of filamin A forms a compact structure that can be extended}

Salla RUSKAMO*1, Robert GILBERT $\uparrow$, Gregor HOFMANN $\uparrow$, Pengju JIANG $§$, lain D. CAMPBELL + , Jari YLÄNNE*2 and UIII PENTIKÄINEN*2

${ }^{*}$ Department of Biological and Environmental Science and Nanoscience Center, University of Jyväskylä, Jyväskylä, Finland, †Division of Structural Biology, University of Oxford, Roosevelt Drive, Oxford OX3 7BN, U.K., †Department of Biochemistry, University of Oxford, South Parks Road, Oxford OX1 3QU, U.K., and §School of Pharmaceutical Engineering and Life Science, Changzhou University, Changzhou, China

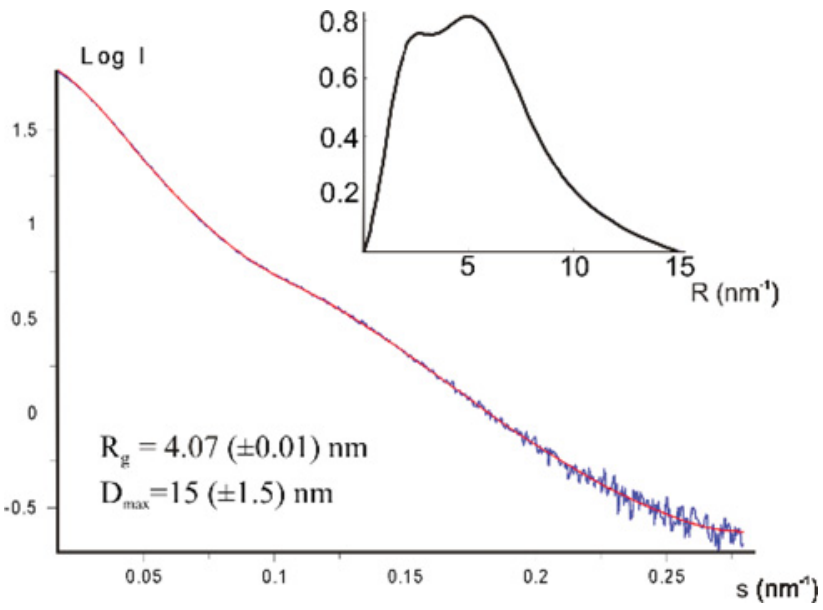

Figure S1 Scattering pattern of FLNa15-21

The experimental scattering pattern of FLNa15-21 is presented as blue and the fit obtained by GASBOR is shown as a red solid line. The distance distribution function of FLNa15-21 calculated by GNOM is shown in the insert.

The cryo-EM reconstructions reported have been deposited in the European Bioinformatics Institute Electron Microscopy Database (http://www.ebi.ac.uk/pdbe/emdb/) under accession codes: EMD-2031 (FLNa16-24, unsymmetrized), EMD-2032 (FLNa16-24 symmetrized), EMD-2033 (FLNa16-24 + $\beta$ peptide, unsymmetrized) and EMD-2034 (FLNa16-24 $+\beta$ peptide, symmetrized)

1 Present address: Department of Biochemistry, University of Oulu, Oulu, Finland.

2 Correspondence may be addressed to either of these authors (email ulla.m.pentikainen@jyu.fi or jylanne@jyu.fi). 
A
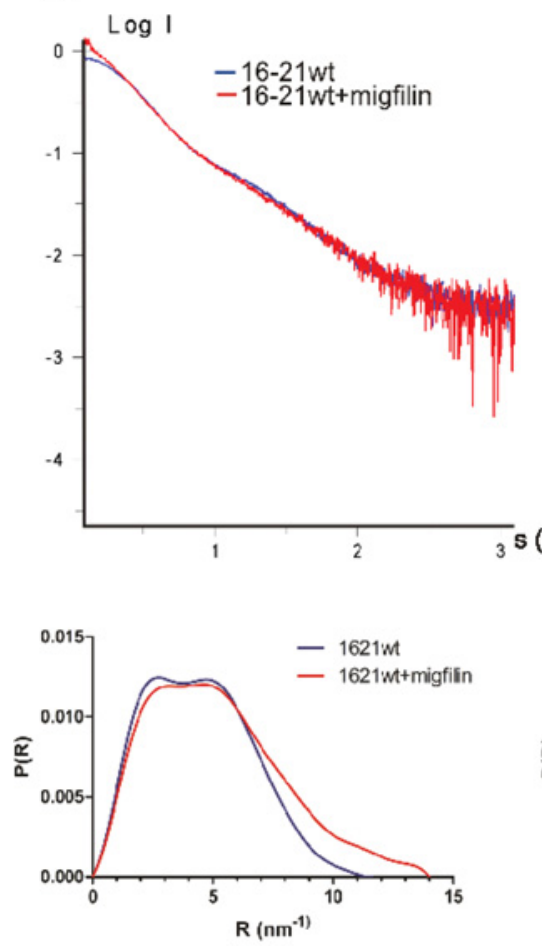

D
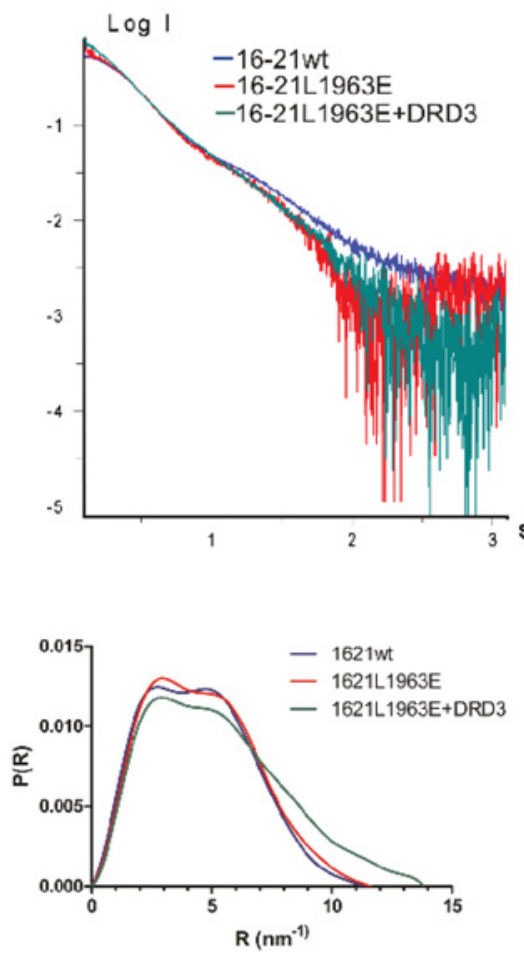

B

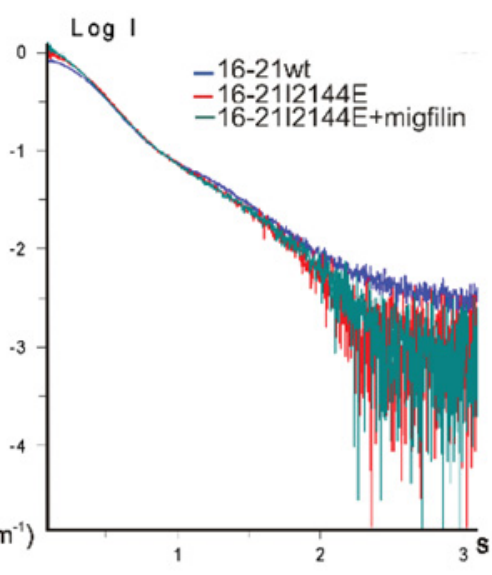

C

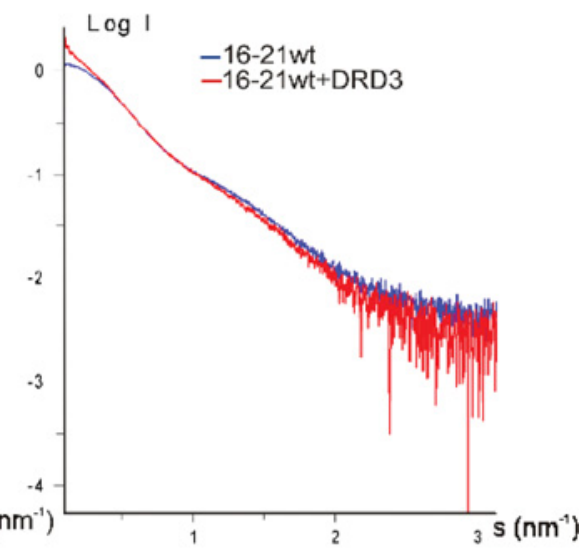

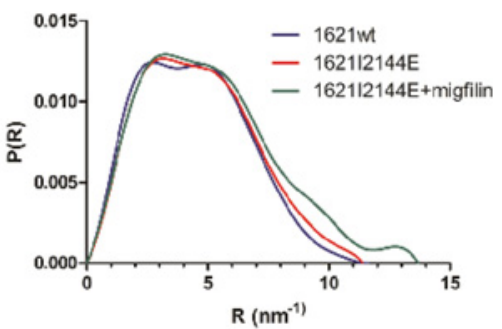

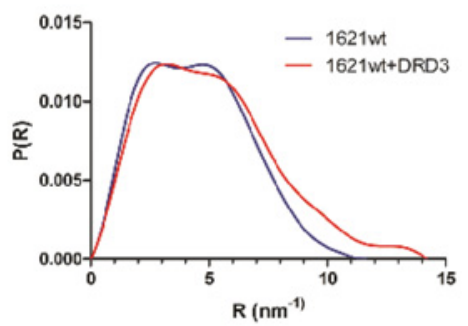

E

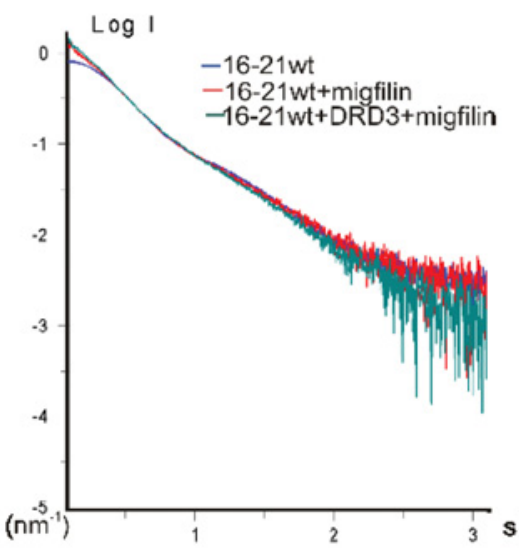

F
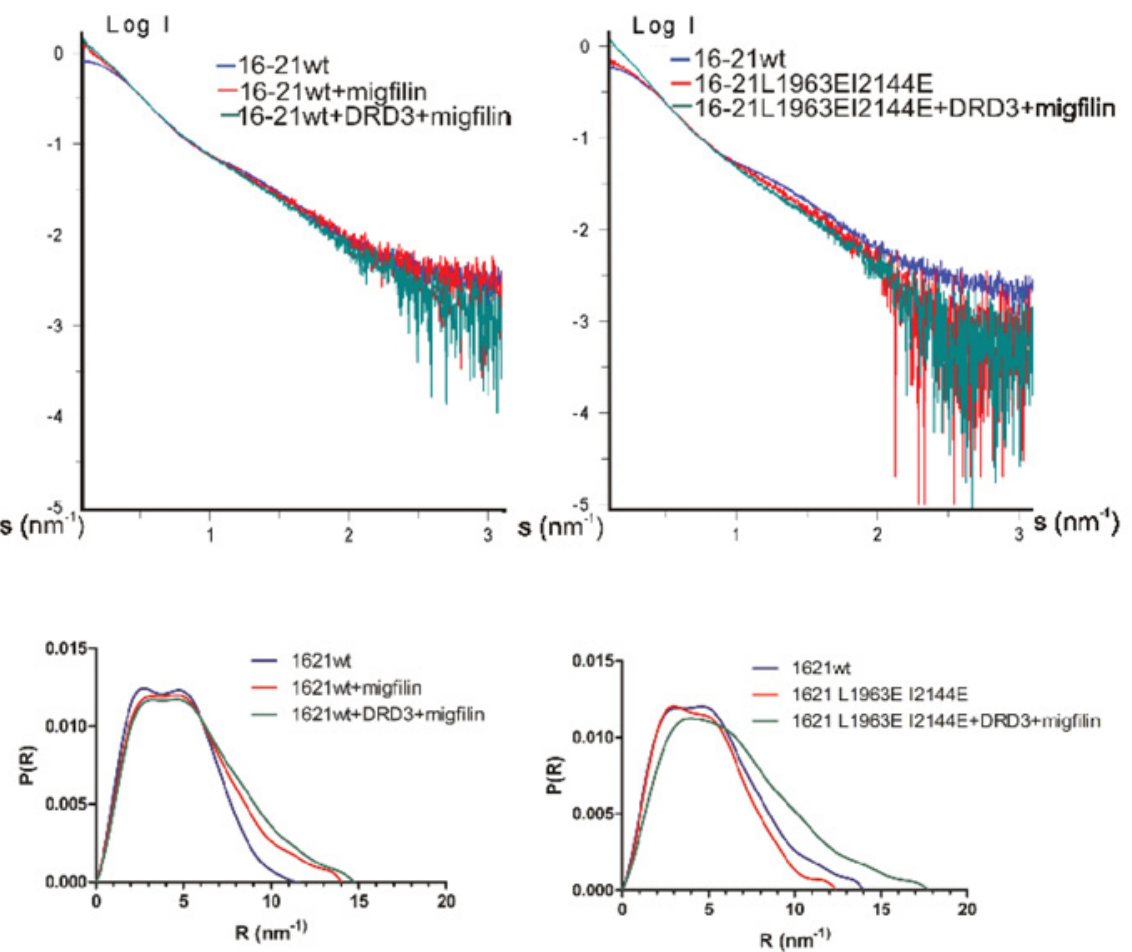

Figure S2 FLNa-binding peptide induced conformational changes in the FLNa16-21 fragment

The corresponding distance distribution functions calculated by GNOM are shown below each scattering curve in corresponding colours. (A) Scattering curves of FLNa16-21 with migfilin peptide (red) and without peptide (blue). (B) Scattering curves of FLNa16-21 (blue), FLNa16-2112144E (red) and FLNa16-2112144E with migfilin peptide (green). (C) Scattering curves of FLNa16-21 with DRD3 peptide (red) and without peptide (blue). (D) Scattering curves of FLNa16-21 (blue), FLNa16-21L1963E (red) and FLNa16-21L1963E with DRD3 peptide (green). (E) Scattering curves of FLNa16-21 (blue), FLNa16-21 with migfilin peptide (red) and FLNa16-21 with both migfilin and DRD3 peptides. (F) Scattering curves of FLNa16-21 (blue), FLNa16-21L1963E 12144E and FLNa16-21L1963E I2144E with both migfilin and DRD3 peptides. 


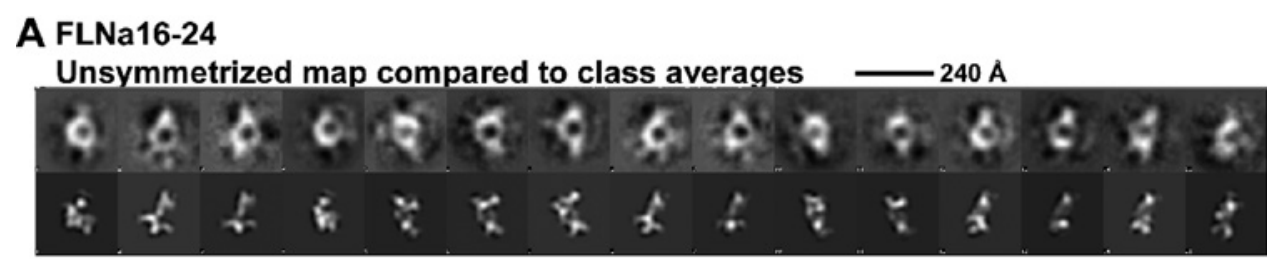

Symmetrized map compared to class averages

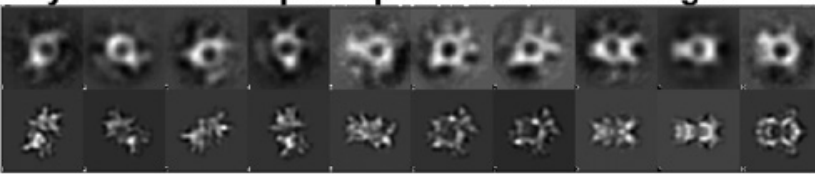

B FLNa16-24 + peptide

Unsymmetrized map compared to class averages

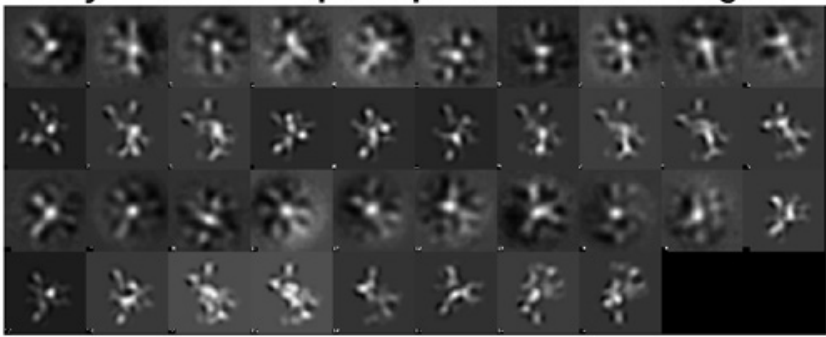

Symmetrized map compared to class averages

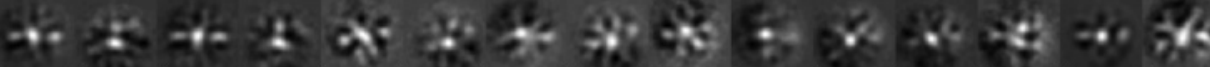

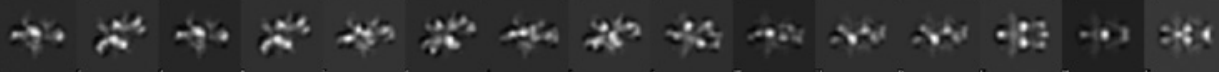

C

FLNa16-24 eigenimages

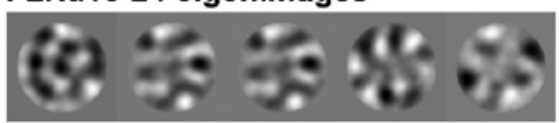

FLNa16-24 + peptide eigenimages

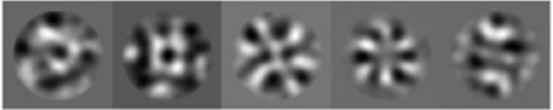

Figure S3 Validation of EM maps

(A) Class averages obtained for FLNa16-21 integrin tail peptide, showing a two-pronged structure, compared with reprojections of the unsymmetrized and symmetrised reconstructions. The upper row shows the class averages, the lower row matching representative reprojections of the reconstructions, in each case. Scale bar $=240 \AA$. (B) As (A), but for FLNa16-21 with peptide. (C) Eigenimages generated during classification of the FLNa16-21 images without and with the peptide present. 

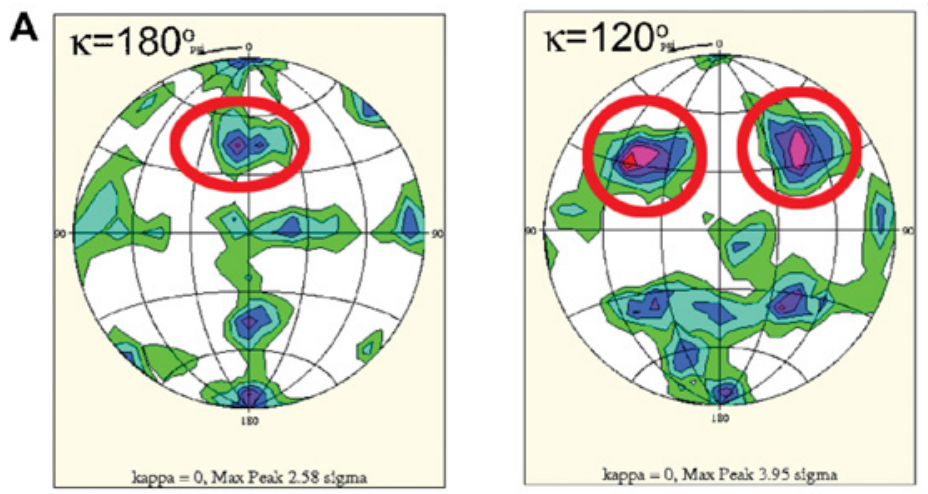

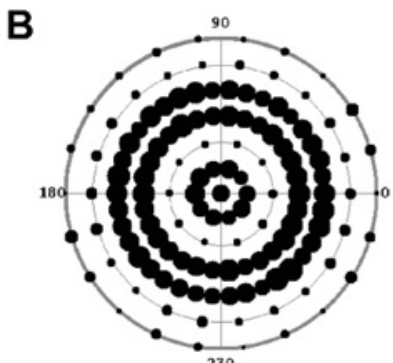

FLNa16-24

D

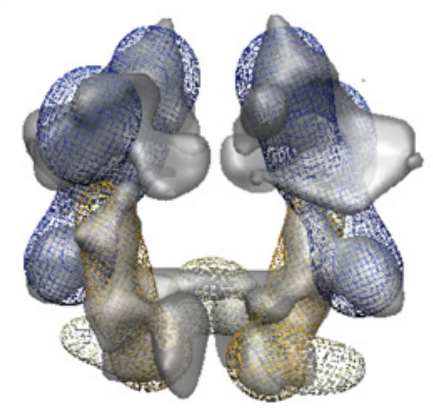

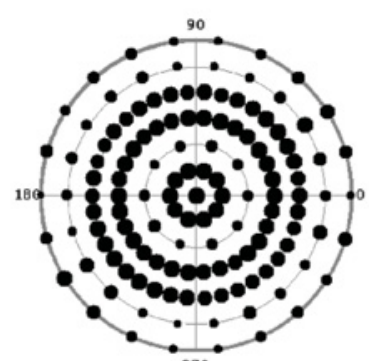

FLNa16-24- $\beta 7$
C

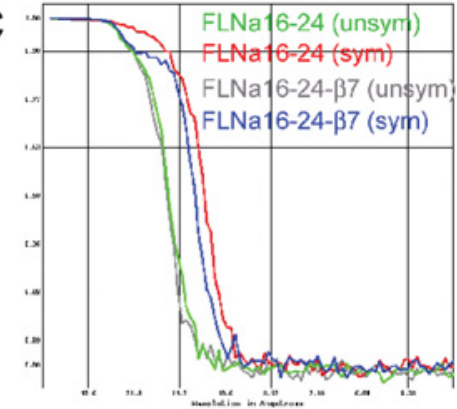

E

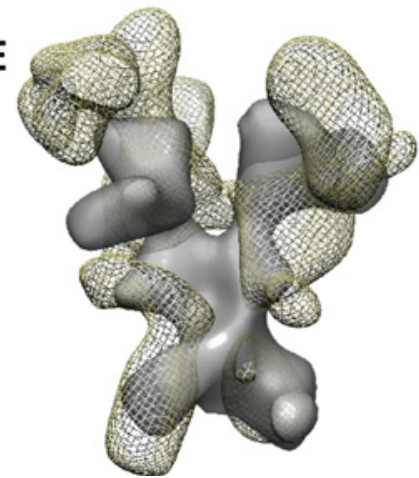

Figure S4 Analysis and comparisons of EM maps

(A) Self-rotation function of the unsymmetrized reconstruction shows 2-fold peaks (left $\kappa=180^{\circ}$ ) and 3-fold peaks (right $\kappa=120^{\circ}$ ). The 2-fold peak on the left-hand side panel and the two 3-fold peaks on the right-hand side panel are ringed in red. Note that the 3-folds sit either side of the 2-fold. (B) Angular distribution plots for the two samples in which the Euler angle at $\theta$ is axial and $\varphi$ circumferential. A relatively even angular distribution is observed, but there is some clumping in orientation distribution, most likely due to the relative flatness of the molecule (see Figure $5 \mathrm{~A}$ of the main text). The size of the plot symbols does not directly correlate with the size of the bins; symbols are shown with a cutoff of 100 particles in each case and an absolute spotsize cutoff of 30 for FLNa16-24 and 20 for FLNa16-24 with peptide. (C) FSC curve for the two unsymmetrized reconstructions: FLNa16-24 in green and FLAa16-24 + $\beta 7$ peptide in grey; and of the two symmetrized reconstructions: FLNa16-24 in red and FLNa16-24 + $\beta 7$ peptide in blue. (D) Fitting of the ab initio SAXS envelope of FLNc23-24 [1] (grey mesh) to the EM map (grey surface). The SAXS envelopes of FLNa16-21 (blue mesh) and FLNa22-23 (yellow mesh) are shown as in Figure 4 of the main text. (E) Superposition of the unsymmetrized FLNa16-24 map without (grey surface) and with (yellow mesh) $\beta 7$ peptide bound. In this Figure the maps are filtered at $30 \AA$.

\section{REFERENCE}

1 Sjekloća, L., Pudas, R., Sjöblom, B., Konarev, P., Carugo, O., Rybin, V., Kiema, T. R., Svergun, D., Ylänne, J. and Djinović Carugo, K (2007) Crystal structure of human filamin C domain 23 and small angle scattering model for filamin C 23-24 dimer. J. Mol. Biol. 368, $1011-1023$

Received 27 February 2012/18 May 2012; accepted 8 June 2012

Published as BJ Immediate Publication 8 June 2012, doi:10.1042/BJ20120361 